\title{
Magnesium lithospermate B protects cardiomyocytes from ischemic injury via inhibition of TAB1-p38 apoptosis signaling
}

\author{
Chang-Sheng Du' ${ }^{1}$, Rui-Fang Yang ${ }^{2}$, Shu-Wei Song ${ }^{2}$, Yi-Ping Wang ${ }^{3}$, Jiu-Hong Kang ${ }^{1}$, Ru Zhang ${ }^{1}$, Ding-Feng Su ${ }^{2 *}$ \\ and $\mathrm{Xin} \mathrm{Xie}^{1,3 *}$
}

\author{
Laboratory of Receptor-Based BioMedicine, School of Life Sciences and Technology, Shanghai Key Laboratory of Signaling and Disease Research, Tongji University, \\ Shanghai, China \\ 2 Department of Pharmacology, School of Pharmacy, Second Military Medical University, Shanghai, China \\ ${ }^{3}$ State Key Laboratory of Drug Research, Shanghai Institute of Materia Medica, Chinese Academy of Sciences, Shanghai, China
}

Edited by:

Tong-Hui Ma, Jilin University, China

Reviewed by:

Hong Yang, Liaoning Normal University,

China

Yuanjian Li, Huanan University of

Science and Technology, China

*Correspondence:

Xin Xie, Shanghai Institute of Materia

Medica, 189 Guo Shou Jing Road,

Shanghai 201203, China.

e-mail:xxie@mail.shcnc.ac.cn and

Ding-Feng Su, Department of

Pharmacology, School of Pharmacy,

Second Military Medical University,

325 Guo-He Road, Shanghai 200433,

China.

e-mail:dfsu2008@gmail.com
Danshen has been used in traditional Chinese medicine for hundreds of years to treat cardiovascular diseases. However, its precise cardioprotective components and the underlying mechanism are still unclear. In the present study, we demonstrated that in a rat model of acute myocardial infarction, the treatment with magnesium lithospermate $B(M L B)$, the representative component of phenolic acids in Danshen, significantly reduced the infarct size and the blood lactate dehydrogenase level. In contrast, tanshinone IIA, the representative component of lipophilic tanshinones in Danshen, had no such protective effects. Moreover, in the simulated ischemia cell model, MLB treatment considerably increased the cell viability and reduced the sub-G1 population and the apoptotic nuclei, indicating its anti-apoptotic effect. Further mechanism study revealed that the ischemia-induced p38 phosphorylation was abolished by MLB treatment. Interestingly, MLB specifically inhibited the TGF $\beta$-activated protein kinase 1-binding protein 1 (TAB1) mediated p38 phosphorylation through disrupting the interaction between TAB 1 and p38, but it did not affect the mitogen-activated protein kinase 3/6 mediated p38 phosphorylation. In conclusion, the present study identifies MLB as an active component of Danshen in protecting cardiomyocytes from ischemic injury through specific inhibition of TAB1-p38 apoptosis signaling. These results indicate TAB1-p38 interaction as a putative drug target in treating ischemic heart diseases.

Keywords: magnesium lithospermate B, ischemia, apoptosis, p38,TAB1

\section{INTRODUCTION}

Danshen, the rhizome of Salvia miltiorrhiza, is one of the famous ancient Chinese herbal medicines and is traditionally believed to "invigorate" the blood and reduce blood clotting. Remedies containing Danshen have been used customarily to treat coronary heart diseases, particularly angina pectoris and myocardial infarction (MI) (Ji et al., 2000). Although Danshen is officially listed in the Chinese Pharmacopoeia and is used widely and successfully in clinics in China, the exact mechanism for its therapeutic basis is poorly understood. Over the last 50 years, the chemical constituents and biological activities of Danshen have been widely studied. To date, more than 60 ingredients have been identified in Danshen, which are mainly divided into water-soluble phenolic acids and lipophilic tanshinones (Wang et al., 2007). Phenolic acids possess antioxidant and anticoagulant activities, whereas tanshinones show antibacterial, antioxidant, and antineoplastic activities. However, the main active component with cardioprotective effects on ischemic heart diseases and the underlying mechanism remain unresolved.

Ischemic heart diseases are characterized by an inadequate blood flow to the heart, which lead to limited availability of glucose and oxygen, delayed clearance of metabolic wastes and eventually massive death of cardiomyocytes. Earlier investigation indicated that cardiac ischemic injury is due to cell necrosis. More recently, apoptosis has been implicated in many forms of cardiac pathology and plays major roles in ischemia-induced cardiomyocytes death (Fliss and Gattinger, 1996; Liu et al., 2009). p38, one of the key factors in the mitogen-activated protein kinase (MAPK) signaling pathway, is activated during simulated ischemia and reperfusion (Ma et al., 1999). Pharmacological inhibition of p38 protects cardiomyocytes from apoptosis during simulated ischemia in vitro (Cheng, 2006). Interestingly, this $\mathrm{p} 38$ phosphorylation and activation are independent of the upstream mitogen-activated protein kinase kinase (MKK) $3 / 6$, but dependent on the autophosphorylation of p38 through its interaction with TGF $\beta$-activated protein kinase 1-binding protein 1 (TAB1) (Ge et al., 2002; Tanno et al., 2003). Thus, inhibition of the TAB1-p38 mediated apoptosis signaling has been implicated to be a potential way for the treatment of ischemic heart diseases.

In the present study, we investigated the protective effects of two representative components of Danshen, magnesium lithospermate B (MLB) and tanshinone IIA, in experimental models of ischemia. We demonstrate that MLB acts as one of the major active components of Danshen in protection of cardiomyocytes from ischemic injury and this is achieved by its specific inhibition of TAB1-p38 apoptosis signaling.

\section{MATERIALS AND METHODS ANIMALS}

Sprague-Dawley (SD) rats were purchased from the Experiment Animal Center, Chinese Academy of Sciences in Shanghai. All animals were maintained under pathogen-free conditions prior to use. 
Male rats weighing 180-220 g were anesthetized with pentobarbital sodium $(50 \mathrm{mg} / \mathrm{kg}$ ) given intraperitoneally before surgery. All animal experiments were in accordance with the National Institutes of Heath Guide for the Care and Use of Laboratory Animals and were approved by Biological Research Ethics Committee, Shanghai Institutes for Biological Sciences, Chinese Academy of Sciences.

\section{RAT ISCHEMIA MODEL}

Rat ischemia model was established by the left anterior descending artery (LAD) ligation as previously described (Connolly et al., 2006; Hu et al., 2009; Wang et al., 2009a). Briefly, after intraperitoneal drug administration, male SD rats were anesthetized and placed in dorsal decubitus supine position and a left thoracotomy was performed at the 5th intercostal space under aseptic surgical conditions. An intercostal retractor was positioned to maintain free access to the heart. The apex of the heart was partially positioned outside the thorax and the LAD was ligated approximately $2 \mathrm{~mm}$ from its origin. The heart was repositioned inside the thorax, which was subsequently closed. The air in the pneumothorax was then gently reduced by external compression. After breathing recovery, the animals were housed individually until the end of the experiment. Sham operated controls were treated in an identical fashion including administration of anesthesia but did not undergo ligature tie off. At the end of LAD ligation, the area at risk and infarct size of the left ventricular tissue sections was assessed by NBT staining as previously described (Zacharowski et al., 1999). The blood samples were collected $1 \mathrm{~h}$ after LAD ligation and serum lactate dehydrogenase level was measured spectrophotometrically using a lactate dehydrogenase assay kit (Shanghai Chemical Reagent Research Institute) according to the manufacturer's instructions.

\section{CELL CULTURES AND REAGENTS}

H9c2 cell (passage 18-25; American Type Culture Collection, Rockville, MD, USA), a clonal line derived from embryonic rat heart, was cultured in DMEM (Gibco-BRL) supplemented with $10 \%(\mathrm{v} / \mathrm{v})$ heat inactivated fetal bovine serum and antibiotics, under an atmosphere of $95 \%$ air and $5 \% \mathrm{CO}_{2}$ at $37^{\circ} \mathrm{C}$. "Danshen Injection" was from Shanghai No.1 Biochemical Pharmacological Company. MLB with $100 \%$ purity and MLB with $83.2 \%$ purity used in cell model and animal models respectively were supplied by Shanghai Institute of Materia Medica, Chinese Academy of Sciences. Other reagents were from Sigma.

\section{SIMULATED ISCHEMIA IN VITRO}

$\mathrm{H} 9 \mathrm{c} 2$ cardiac myocytes were exposed to simulated ischemia as described (Fiedler et al., 2006; Wang et al., 2009b). In brief, the cells were switched from maintenance medium to an ischemic buffer and were incubated in a hypoxia chamber flushed with $5 \% \mathrm{CO}_{2}$ and $95 \% \mathrm{~N}_{2}$ for indicated period of time. Control cells were cultured in a normal buffer and incubated at $37^{\circ} \mathrm{C}$ in an atmosphere containing $5 \% \mathrm{CO}_{2}$ and $95 \%$ room air (normoxia).

\section{IMMUNOFLUORESCENCE MICROSCOPY}

Heart tissue sections were incubated with primary antibodies against total p38 or phosphorylated p38 and subsequently with an FITC-conjugated secondary antibody. Images were acquired with a confocal fluorescence microscope (Leica TCS SP2) as previously described (Kang et al., 2005).

\section{FACS}

The cells were harvested and washed with PBS ( $\mathrm{pH} 7.2)$ twice and suspended in $80 \%$ ethanol at $-20^{\circ} \mathrm{C}$ for $24 \mathrm{~h}$. The cells were then thawed at room temperature, washed twice with PBS and resuspended in the extraction buffer $\left(0.2 \mathrm{M} \mathrm{Na}_{2} \mathrm{HPO}_{4}, 0.1 \mathrm{M}\right.$ citric acid) for $5 \mathrm{~min}$. The cells were stained with $50 \mu \mathrm{g} / \mathrm{ml}$ propidium iodide in PBS containing RNase A $(100 \mu \mathrm{g} / \mathrm{ml})$ for $30 \mathrm{~min}$. The cell cycle distribution and quantitation of apoptotic cells were measured by a FACS calibur flow cytometer (Becton-Dickinson) (Du et al., 2004).

\section{MTS ASSAY}

The viability of cultured cells was evaluated by MTS assay (Promega) as previously described (Jing et al., 1999). Cells were seeded onto the 96-well plates at a density of 5000-10,000 cells/100 $\mu \mathrm{l} /$ well overnight. After treatment of various reagents, the cells were subjected to simulated ischemia for indicated time period. $20 \mu \mathrm{l}$ of MTS solution was then added and cells were incubated at $37^{\circ} \mathrm{C}$ for $4 \mathrm{~h}$. Absorbance at $490 \mathrm{~nm}$ was measured using a microplate reader.

\section{TERMINAL DEOXYNUCLEOTIDYL TRANSFERASE dUTP NICK END-LABELING (TUNEL)}

TUNEL was applied to detect DNA fragmentation that results from apoptotic signaling cascades. Cells were grown on glass cover slips for $24 \mathrm{~h}$. Cells were then subjected to simulated ischemia for indicated time period and fixed with $4 \%$ polyformaldehyde. Cells were washed and permeabilized with $0.2 \%$ Tween in PBS containing $0.2 \%$ BSA. After fluorescein staining with TdT end-labeling cocktail (TdT buffer, Biotin-dUTP, and TdT) and avidin-FITC, DNA fragmentation was analyzed on a fluorescence microscope. Analysis of tissue samples was performed according to the manufacturer's instructions (Upstate).

\section{WESTERN BLOT ANALYSIS}

Proteins were denatured, resolved on SDS-PAGE gels, and transferred onto nitrocellulose membranes. Primary antibodies were added at the recommended dilutions. Detection was performed with IRDyeTM800CW-conjugated secondary antibody and the infrared fluorescence image was obtained using the Odyssey infrared imaging system (Li-Cor Bioscience). In some experiments, protein bands were visualized by enhanced chemiluminescence.

\section{IMMUNOPRECIPITATION AND IN VITRO p38 ACTIVITY ASSAY}

After simulated ischemia, cells or homogenized heart tissues were lysed with ice cold immunoprecipitation buffer. Cell lysates were incubated with a total p38 antibody overnight at $4^{\circ} \mathrm{C}$ and then precipitated with protein A agarose beads for $2 \mathrm{~h}$ at $4^{\circ} \mathrm{C}$. The beads were washed five times with lysis buffer and recombinant activating transcription factor-2 (ATF-2) fusion protein was added.p38 activity in immunoprecipitates was assessed by Western blot analysis of phospho-transcription factor-2 (Jing et al., 1999; Bonta et al., 2009).

\section{STATISTICAL ANALYSIS}

Results of the experiments were expressed as means \pm S.E.M. Student $t$-test was performed for the statistical analysis of results. For all tests, a $P$-value less than 0.05 was considered statistically significant. 


\section{RESULTS}

\section{MLB TREATMENT ATTENUATES ISCHEMIC HEART INJURY IN RAT}

In order to test the protective effect of Danshen in myocardial ischemic injury, rat ischemia model was established by $1 \mathrm{~h} \mathrm{LAD}$ ligation. "Danshen Injection," a total extraction from Danshen ( Wu et al., 2007), MLB and tanshinone IIA, two representative components of Danshen, were applied just before the surgery at a dosage normalized to their natural composition in the plant (Figure 1). Compared to normal saline treated group, the heart infarct sizes in "Danshen Injection" and MLB $(10 \mathrm{mg} / \mathrm{kg})$ treated rats were reduced by $24.5 \%$ and $23.6 \%$ respectively, which is more effective than the control with a first-line drug nitroglycerin $(30 \mathrm{mg} /$ $\mathrm{kg}$, infarct size reduction about 15\%) (Figures 1A,B). In contrast, tanshinone IIA had no effect on reducing the infarct size. The reduction of the infarct area by MLB treatment was very significant and the differences can be clearly observed by visual examination (Figure 1C). The heart area at risk of acute MI was comparable between the normal saline group and drug treatment groups (data not shown). Blood samples were collected after $1 \mathrm{~h} \mathrm{LAD}$ ligation and blood lactate dehydrogenase level, another marker for heart impairment during the MI (Garlid et al., 1997), was found to increase by 2.1-folds in the normal saline treatment group compared to the sham operation group. This increase was inhibited by MLB or "Danshen Injection" treatment but not by tanshinone IIA (Figure 1D). These data demonstrate that MLB acts as one of the major active components of Danshen in protection of cardiomyocytes from ischemic injury.

\section{MLB TREATMENT INHIBITS APOPTOSIS IN ISCHEMIC CELL MODEL AND RAT HEART}

It has been demonstrated that cell apoptosis is one of the major causes of cardiomyocyte injury during ischemic insults (Fliss and Gattinger, 1996). So we tested the ability of MLB in inhibiting ischemic induced apoptosis. H9c2 is a cardiac myoblasts cell line that is widely used to study the ischemic myocardial injuries in vitro (Bonavita et al., 2003; Agnetti et al., 2005). TUNEL assay revealed that simulated ischemia triggered apoptosis in about 35\% of $\mathrm{H} 9 \mathrm{c} 2$ cells. But in $10 \mu \mathrm{M}$ and $30 \mu \mathrm{M}$ MLB treatment groups, cell apoptosis was remarkably reduced to $22 \%$ and $12 \%$, respectively (Figures 2A,B). This protection effect of MLB on H9c2 apoptosis was further validated by the FACS analysis. The percentage of sub-G1 cells were commonly used as a measure of apoptosis. Results indicated, at both 10 and $30 \mu \mathrm{M}$ concentrations, MLB significantly reduced the sub-G1 population of $\mathrm{H} 9 \mathrm{c} 2$ cells in simulated ischemia (Figure 2C). Moreover, TUNEL staining revealed cardiomyocytes apoptosis in the infarcted rat hearts was also inhibited by MLB treatment (Figure 2D), supporting the cardioprotection role of MLB in vivo.

\section{MLB INHIBITS ISCHEMIA-INDUCED APOPTOSIS BY INHIBITING p38 PHOSPHORYLATION AND ACTIVATION}

MAPKs play important roles in the ischemic injury of cardiomyocytes (Armstrong, 2004). In order to explore the potential signaling pathways involved in MLB-mediated cardioprotection, the H9c2 cells were pretreated with three types of MAPK inhibitors
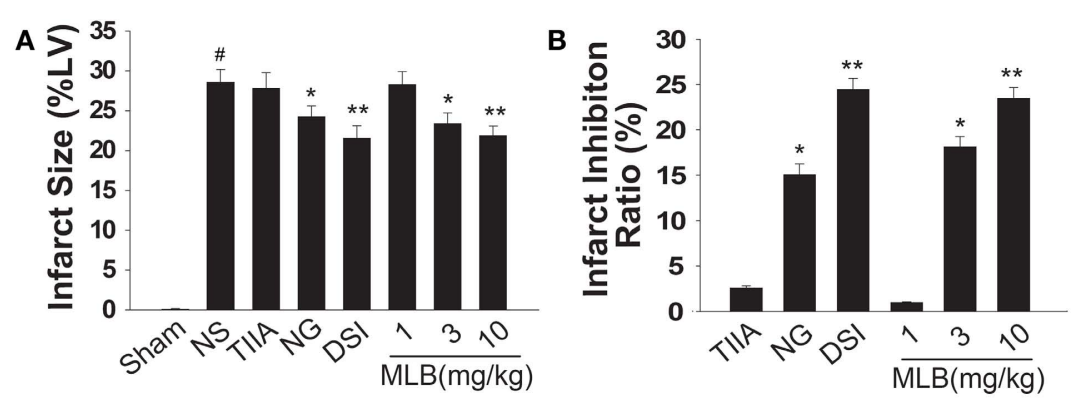

C

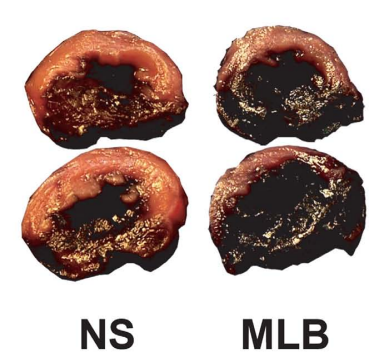

FIGURE 1 | MLB treatment attenuates ischemic injury in rat myocardial infarction (MI) animal model. (A) Rats were intraperitoneally injected with normal saline (NS), tanshinone IIA (TIIA, $1 \mathrm{mg} / \mathrm{kg}$ ), nitroglycerin (NG, $30 \mathrm{mg} / \mathrm{kg}$ ), "Danshen Injection" (DSI, $120 \mu \mathrm{l} / \mathrm{kg}$ ) and MLB (1-10 mg/kg), before LAD ligation. Heart infarct size was assessed after $1 \mathrm{~h}$ global ischemia by LAD ligation as a percentage of total left ventricular weight (\%LV). (B) Infarct inhibition ratio by
D

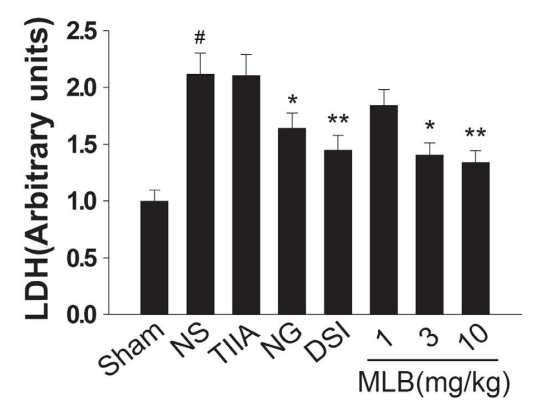

medicine treatment is depicted as a percentage of reduced infarct size compared to the infarct size in NS group. (C) Representative pictures of NS or MLB (10 mg/kg) treated rat MI hearts (red indicates the infracted area). (D) Lactate dehydrogenase (LDH) level in animal blood samples from (A). $n=8 \sim 10 ; \#, P<0.001$ compared with sham group. ${ }^{*} P<0.05$; ${ }^{*} P<0.01$ compared with NS group. 

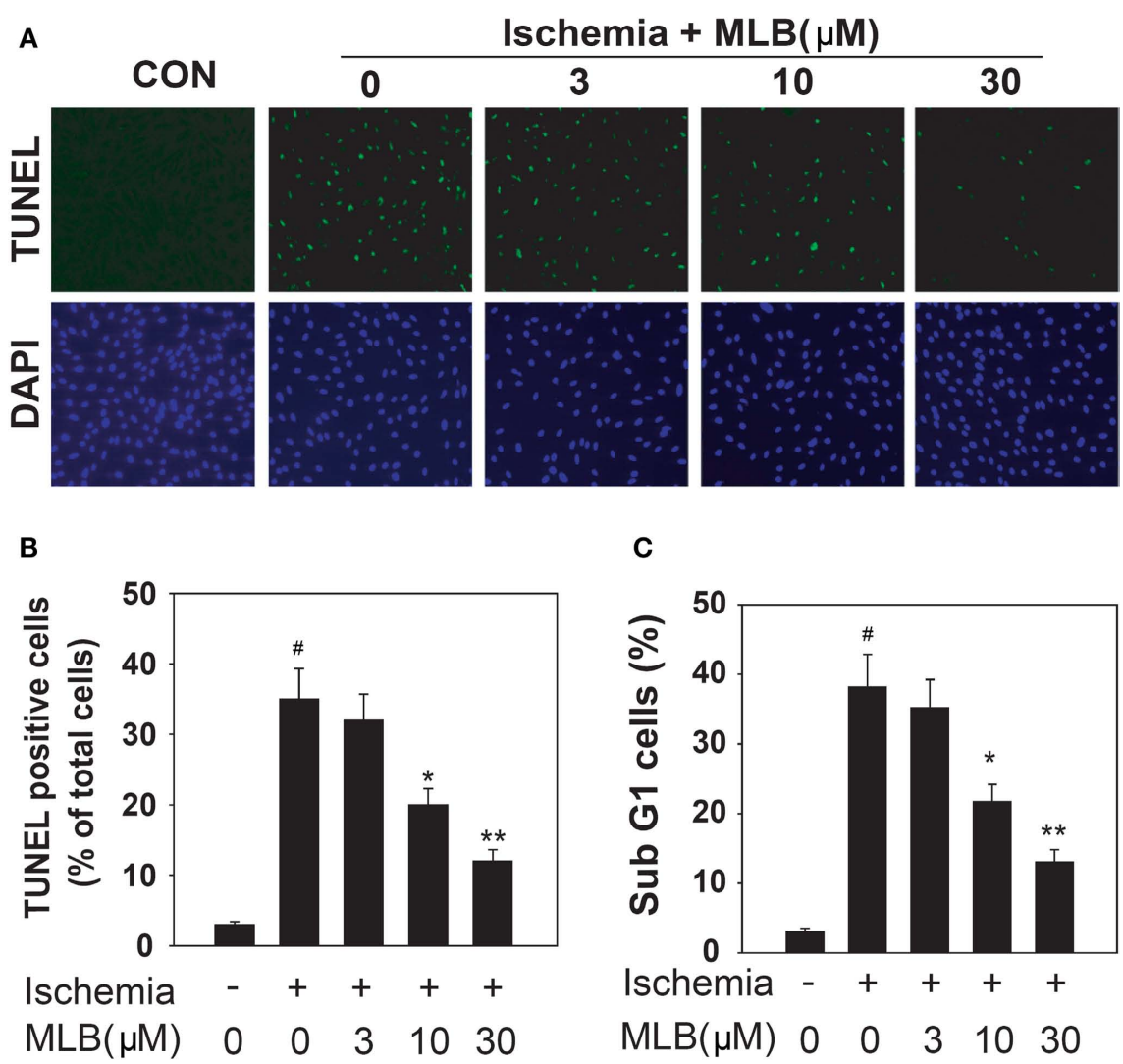

C
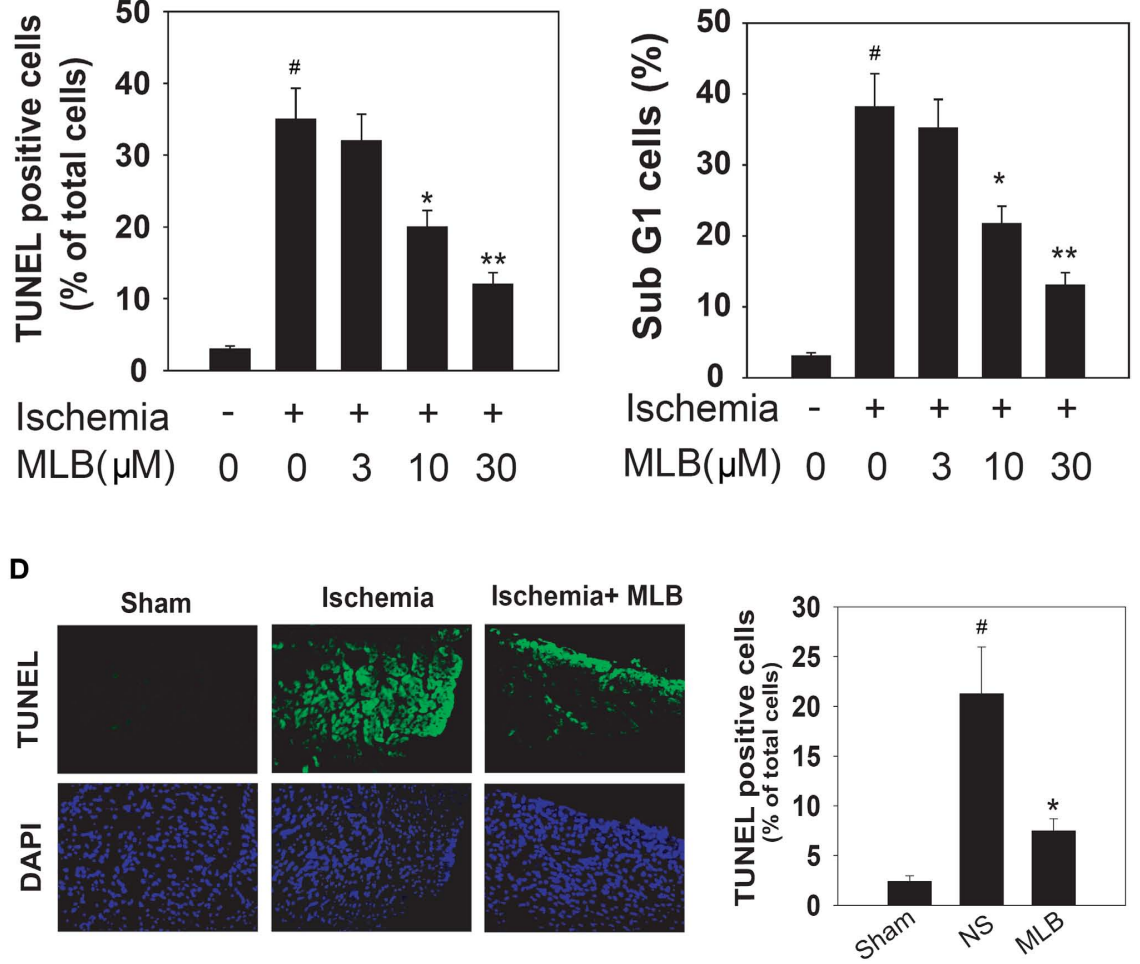

FIGURE 2 | MLB inhibits apoptosis in simulated ischemia treated H9c2 cells and ischemic rat hearts. (A) H9c2 cells were pretreated with NS or MLB (3-30 $\mu \mathrm{M}$ ) for $30 \mathrm{~min}$ and subjected to simulated ischemia (16 h). Cell apoptosis was assessed by TUNEL assay. The mock treated cells were shown as control (CON). DAPI staining was used to reveal the total cell number. (B) Quantitative presentation of the TUNEL analysis shown in (A). Values are shown as a percentage of the TUNEL positive cell number to the total cell number. (C) Cell apoptosis assessed by FACS analysis. Cells were stained with propidium iodide and apoptosis was measured as the percentage of sub-G1 cells. (D) TUNEL assay was performed in rat hearts subjected to $24 \mathrm{~h} \mathrm{LAD}$ ligation. Animals were pretreated with NS or MLB $(10 \mathrm{mg} / \mathrm{kg})$. Data shown were pooled from three independent experiments. $\#, P<0.001$ compared with sham group. ${ }^{*} P<0.05 ;{ }^{*} P<0.01$ compared with NS group. before simulated ischemia treatment. Interestingly, neither ERK inhibition by PD98059 nor JNK inhibition by SP600125 prevented cell death induced by ischemic injury (Figure 3A). However, p38 inhibition by SB203580 significantly increased cell viability to a similar extent as observed in MLB treatment. Furthermore, when cells were treated with MLB and SB203580 simultaneously, no additive or synergistic protection effects were observed. Cell apoptosis analyzed by TUNEL and FACS supports the MTS results (Figures 3B,C). These results suggest that MLB might exert its cardioprotective effect by inhibiting p38 signaling pathway. Western blot analysis was carried out to further validate this hypothesis. As shown in Figures 3D,E, pretreatment with MLB dose-dependently blocked ischemia-induced elevation of phosphorylated p38 level. In contrast, MLB had no effect on ischemiainduced ERK and JNK phosphorylation (Figures 3D,E). Similar results were obtained when infarcted rat hearts were subjected to Western blot analysis (Figures 4A,B). MLB significantly reduced ischemia-induced p38 phosphorylation but did not affect ERK 

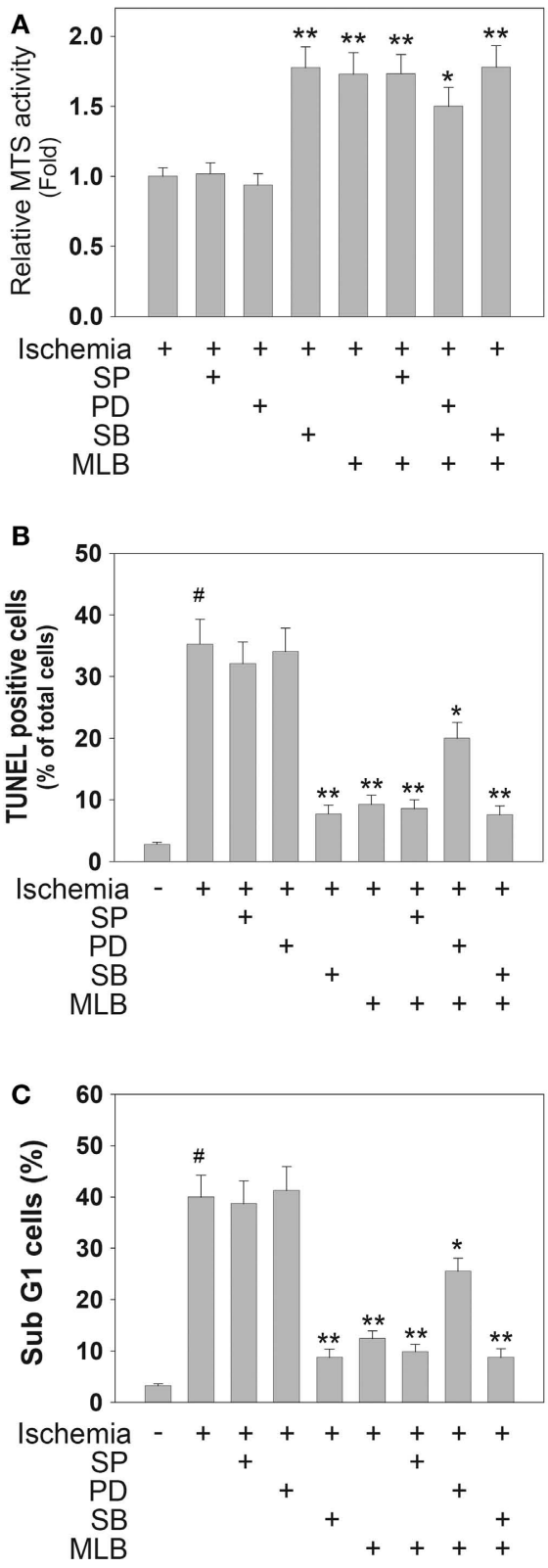

FIGURE 3 | MLB inhibits simulated ischemia-induced apoptosis by inhibiting $\mathbf{p} 38$ phosphorylation in $\mathbf{H 9 c} 2$ cells. (A-C) H9c2 cells were pretreated with $10 \mu \mathrm{M}$ SP600125 (SP, JNK inhibitor), $10 \mu \mathrm{M}$ PD98059 (PD, ERK inhibitor), $1 \mu \mathrm{M} \mathrm{SB} 203580$ (SB, p38 inhibitor) and $30 \mu \mathrm{M}$ MLB for $30 \mathrm{~min}$ and then subjected to simulated ischemia for $16 \mathrm{~h}$. (A) Cell viability was determined by MTS assay. (B,C) Cell apoptosis was assessed by TUNEL assay and FACS analysis. (D) For Western blot analysis, H9c2 cells were pretreated with $30 \mu \mathrm{M}$
D
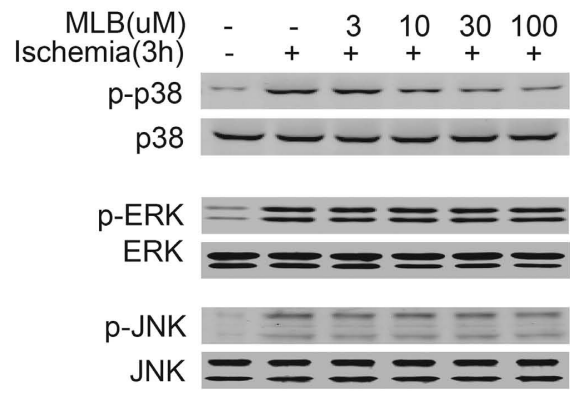

actin

E

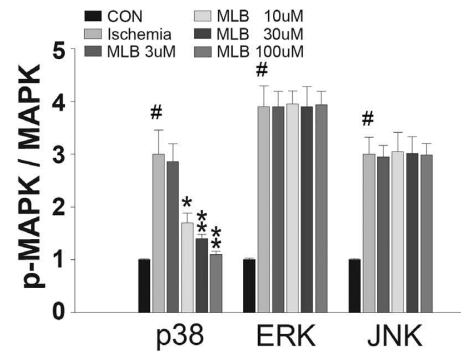

and JNK phosphorylation in rat hearts. Immunofluorescence staining of phosphorylated p38 in infarcted rat hearts clearly demonstrated that MLB treatment dramatically reduced p38 phosphorylation (Figures 4C,D).

It has been established that p38 is activated by dual phosphorylation at ${ }^{180} \mathrm{Thr}$ and ${ }^{182} \mathrm{Tyr}$ residues (Jing et al., 1999), and the phosphorylation of $\mathrm{p} 38$ has been widely used to represent its activation.
Direct measurement of p38 kinase activity was also carried out with immunoprecipitated $\mathrm{p} 38$ from ischemic rat hearts and recombinant ATF-2 was used as a substrate (Figures 4E,F). In agreement with our previous observation, ischemia clearly stimulated p38 phosphorylation as well as the phosphorylation of its substrate ATF2, whereas MLB inhibited this phosphorylation and therefore the activity of $\mathrm{p} 38$. 


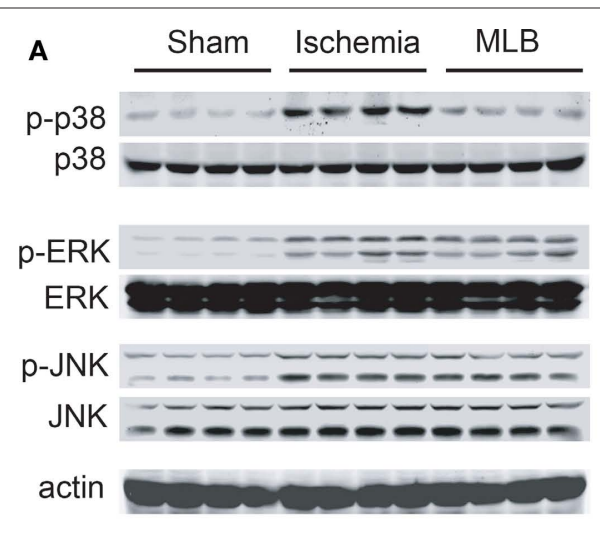

C

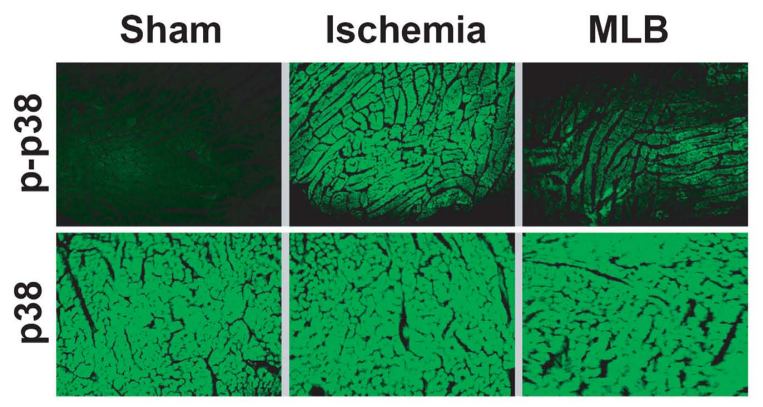

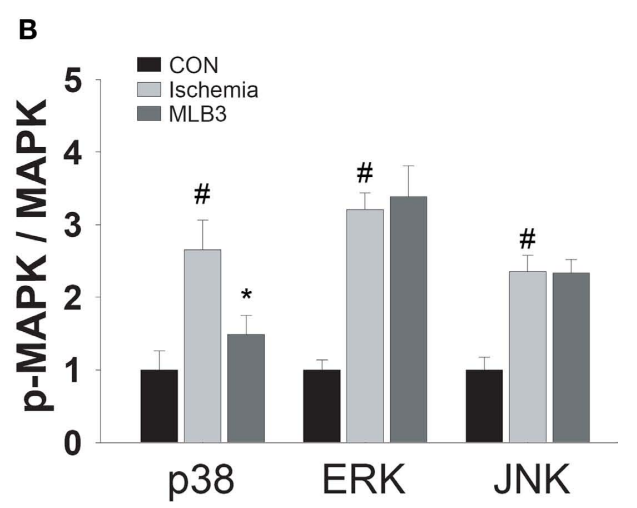

D

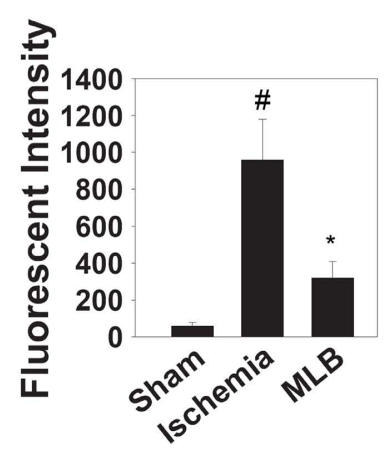

E

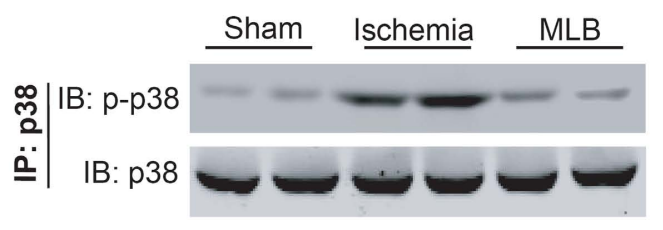

F p-ATF2

ATF2

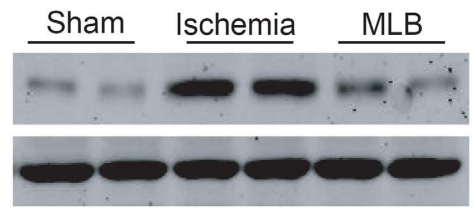

FIGURE 4 | MLB inhibits p38 phosphorylation and activation in ischemic rat heart. MAPKs phosphorylation in ischemic (1 h) rat heart tissues were analyzed by Western blot (A) or immunofluorescence assay (C). Antibodies against total and phosphorylated MAPKs (p38, ERK, JNK) were applied. Detection of actin served as a loading control. Quantitative analysis of Western blot was performed with Scnlmage software (B). Quantification of immunofluorescence was performed with Image-pro software (D). Data shown are pooled from three independent experiments. (E,F) Whole rat hearts were homogenized $1 \mathrm{~h}$ after LAD ligation, and total p38 was immunoprecipitated with an anti-p38 antibody. Phosphorylated p38 and total p38 were analyzed by Western blot (E). P38 kinase activity was assessed in vitro by its ability to phosphorylate ATF-2 (F), \#, $P<0.05$ compared with sham group. ${ }^{*} P<0.05$ compared with ischemia treatment group.

\section{MLB INHIBITS ISCHEMIC INDUCED p38 PHOSPHORYLATION AND ACTIVATION BY DISRUPTING TAB1-p38 INTERACTION}

In the MAPK activation cascade, p38 is generally phosphorylated and activated by MKK3/6 (Chang and Karin, 2001). In order to illustrate whether MKK3/6 is involved in the phosphorylation inhibition of p38 by MLB, sorbitol and TNF, which were reported to induce MKK3/6-dependent p38 phosphorylation (Wysk et al., 1999; Tanno et al., 2003), were applied. Strikingly, MLB treatment did inhibit the ischemia stimulated p38 phosphorylation but not sorbitol or TNF-stimulated p38 phosphorylation (Figure 5A). Similar results were obtained with SB203580, an inhibitor of p38 signaling which specifically inhibits ischemia-induced p38 phosphorylation, but not MKK3/6-mediated p38 phosphorylation (Gum et al., 1998). These findings suggest that MLB might function through a nonMKK3/6 pathway to interfere the p38 signaling.
Recently, a MKK3/6-independent model of p38 activation has been described where the scaffold protein TAB1 promotes $\mathrm{p} 38$ autophosphorylation through interaction with p38 (Ge et al., 2002). This TAB1 mediated p38 phosphorylation and activation has been demonstrated to play important roles in ischemia-induced heart injuries (Ge et al., 2002; Tanno et al., 2003; Li et al., 2005; Fiedler et al., 2006). Therefore, we tested the possibility whether MLB inhibits ischemia-induced p38 phosphorylation by interfering TAB1-p38 interaction. Consistent with the previous report (Tanno et al., 2003), we observed that ischemia (3 h) markedly stimulated p38 phosphorylation and its interaction with TAB1 in H9c2 cells (Figures 5B,C, lane 4). In contrast, TAB1-p38 interaction was not observed in $\mathrm{H} 9 \mathrm{c} 2$ cells treated with sorbitol or TNF (data not shown), indicating that TAB1-p38 interaction is not regulated by $\mathrm{MKK} 3 / 6$ signaling. Interestingly, the TAB1-p38 


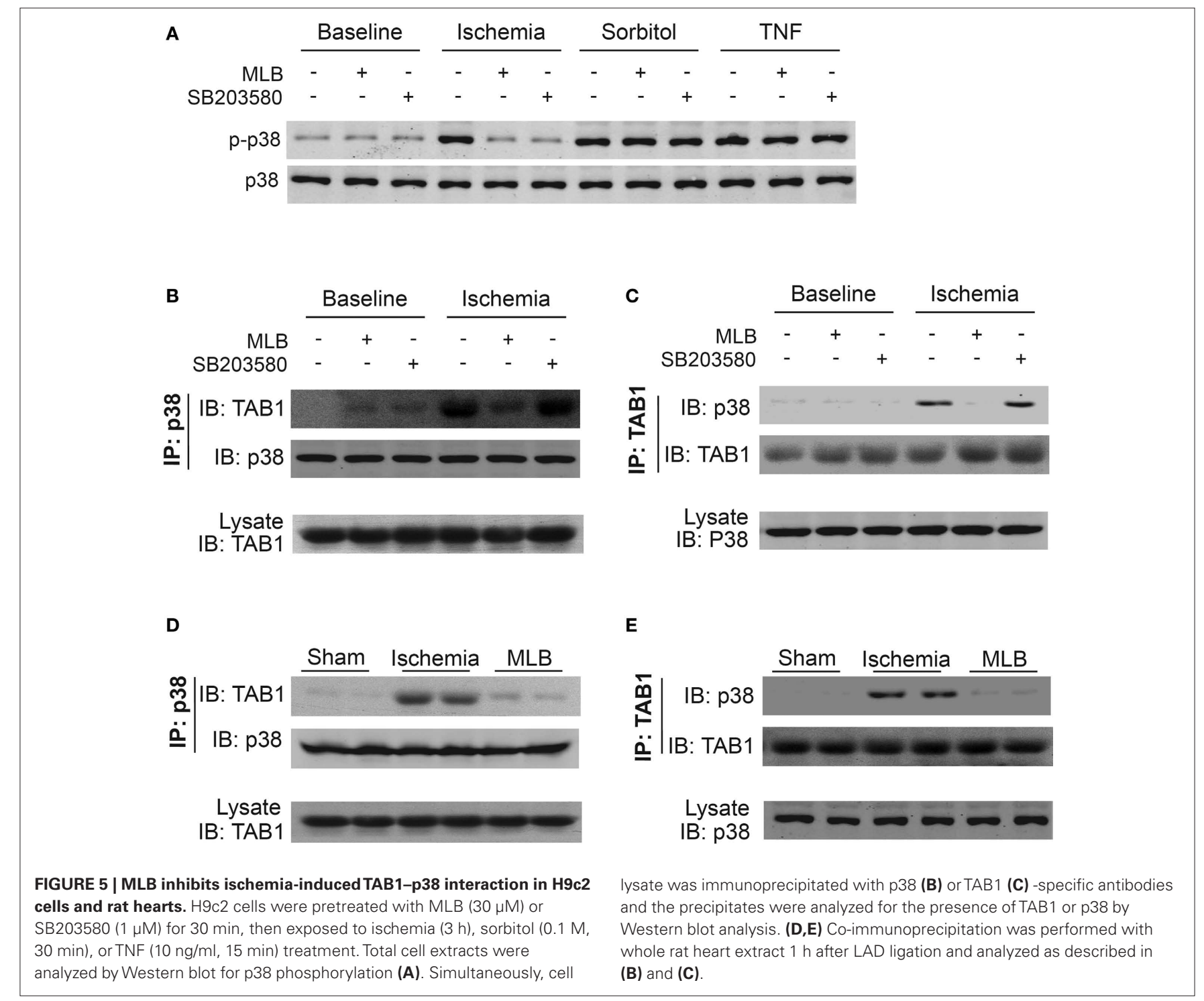

interaction stimulated by ischemia was almost completely disrupted by MLB treatment (Figures 5B,C, lane 5). In contrast, SB203580, which inhibits p38 activity through binding to its kinase activity domain, had little influence on the interaction (Figures 5B,C, lane 6). We next examined whether MLB is also able to reduce TAB1-p38 interaction caused by MI in rat heart. As shown in Figures 5D,E, ischemia remarkably enhanced the interaction between p38 and TAB1, which was disrupted by MLB.

Taken together, our results demonstrate that MLB, one of the major active components of Danshen, protects myocardial injuries from ischemic insults in both animal and cell models. This protective effect of MLB is related to its ability to specifically inhibit ischemia-induced TAB1-p38 apoptosis signaling pathway.

\section{DISCUSSION}

Danshen has been widely used in China and, to a lesser extent, in Japan, the United States, and other European countries for the treatment of cardiovascular and cerebrovascular diseases. It has also been indicated for chronic hepatitis and liver fibrosis treatment (Liu et al., 2000), as well as inflammatory diseases such as lethal sepsis (Wan et al., 2006). The chemical constituents of Danshen are divided mainly into water-soluble phenolic acids and lipophilic tanshinones (Wang et al., 2007). The phenolic acids have diversed biological activities including antioxidation, anti-blood coagulation, vasodilation, cell protection, etc. Recent research revealed additional anti-tumor activity and anti-HIV activity of the phenolic acids (Jiang et al., 2005). The lipophilic tanshinones are also found to possess a variety of pharmacological activities such as antibacteria, antioxidation, anti-inflammatory and anti-tumor formation.

In the present study we demonstrate that MLB, the representative component of the water soluble phenolic acids in Danshen, has similar cardioprotective activity in the rat model of ischemia compared to the crude Danshen extract when used at a normalized dosage. In contrast, the tanshinone IIA has little effect when applied at a dosage comparable to its concentration within crude Danshen extract. Therefore, MLB, the representative of phenolic 
acids in Danshen, acts as the major effective component in the treatment of ischemic heart diseases. However, since Danshen has been used to treat many other cardiovascular diseases, tanshinone IIA as well as other components might function under different mechanisms that remain to be investigated.

Apoptosis has been demonstrated as one of the major causes of myocardial injury during ischemia (Thompson et al., 1995; Rodriguez et al., 2002; Garg et al., 2003). It is controlled by a network of signaling pathways, such as MAPKs, PI3K and Akt pathways. The classical MAPK signaling pathway is comprised of three major branches named ERK, JNK, and p38 (Chang and Karin, 2001). All three MAPK cascades have been directly implicated in regulating aspects of cardiomyocytes apoptosis and p38 pathway plays an active role in ischemia-induced cardiomyocytes apoptosis (Ma et al., 1999). Coincidently, our results herein showed that p38, but not the ERK and JNK inhibition, is involved in the MLBmediated cardiac protection. Although PI3K and AKT signaling activation were reported to have cardioprotective effects (Kim et al., 2006). Our preliminary results show that MLB treatment has little influence on this signaling pathway (data not shown). If the specific inhibition of $\mathrm{p} 38$ by MLB is the underlying mechanism of Danshen's therapeutic efficacy, it is worthy to further explore the potential application of specific p38 inhibitors in the treatment of ischemic heart diseases.

P38 is generally activated by the transphosphorylation of its upstream MKK3/6 (Chang and Karin, 2001). However, recent observations indicate that MKK3/6 is not the only mediator of $\mathrm{p} 38$ phosphorylation in vivo. TAB1, a scaffold protein, also promotes p38 autophosphorylation through interaction with p38 (Ge et al., 2002; Tanno et al., 2003). TAB1-mediated p38 activation has been pointed out to play important roles in ischemia-induced myocardial injury (Ge et al., 2002; Tanno et al., 2003; Li et al., 2005; Fiedler

\section{REFERENCES}

Agnetti, G., Maraldi, T., Fiorentini, D., Giordano, E., Prata, C., Hakim, G., Muscari, C., Guarnieri, C., and Claudio Marcello Caldarera, M. C. (2005). Activation of glucose transport during simulated ischemia in $\mathrm{H} 9 \mathrm{c} 2$ cardiac myoblasts is mediated by protein kinase C isoforms. Life Sci. 78, 264-270.

Armstrong, S. C. (2004). Protein kinase activation and myocardial ischemia/ reperfusion injury. Cardiovasc. Res. 61, 427-436.

Bonavita, F., Stefanelli, C., Giordano, E., Columbaro, M., Facchini, A., Bonafe, F., Caldarera, C. M., and Guarnieri, C. (2003). H9c2 cardiac myoblasts undergo apoptosis in a model of ischemia consisting of serum deprivation and hypoxia: inhibition by PMA. FEBS Lett. 536, 85-91.

Bonta, P. I., and de Vries, C. J. (2009). Hypoxia regulates resistin in vascular smooth muscle cells, what next? J. Hypertens. 26, 2349-2360.

Chang, L., and Karin, M. (2001). Mammalian MAP kinase signalling cascades. Nature 410, 37-40.
Cheng, T. O. (2006). Danshen: a versatile Chinese herbal drug for the treatment of coronary heart disease. Int. J. Cardiol. 113, 437-438.

Connolly, E. P., Thuillier, V., Rouy, D., Bouetard, G., and Schneider, R. J. (2006). Inhibition of cap-initiation complexes linked to a novel mechanism of eIF4G depletion in acute myocardial ischemia. Cell Death Differ. 13, 1586-1594.

Du, C., Zhang, Q., Li, C., Miao, D., and Gui, J. (2004). Induction of apoptosis in a carp leucocyte cell line infected with turbot (Scophthalmus maximus L.) rhabdovirus. Virus Res. 101, 119-126.

Fiedler, B., Feil, R., Hofmann, F., Willenbockel, C., Drexler, H., Smolenski, A., Lohmann, S. M., and Wollert, K. C. (2006). cGMP-dependent protein kinase type I inhibits TAB1-p38 mitogen-activated protein kinase apoptosis signaling in cardiac myocytes. J. Biol. Chem. 281, 32831-32840.

Fliss, H., and Gattinger, D. (1996). Apoptosis in ischemic and reperfused

et al., 2006). Consistent with these reports, we demonstrated that MLB treatment disrupts ischemia stimulated TAB1-p38 interaction in vivo. In vitro co-immunoprecipitation experiments revealed that MLB can not directly inhibit the TAB1-p38 interaction (data not shown), suggesting that TAB1-p38 upstream signal pathways are involved in the MLB activity. Previous reports showed that both PKGI (cGMP-dependent protein kinase type I) and AMPK (AMP-activated protein kinase) are involved in the regulation of TAB1-p38 interaction in the ischemic heart (Li et al., 2005; Fiedler et al., 2006). Whether MLB modulates TAB1-p38 interaction via PKGI and/or AMPK requires further investigation.

In summary, the present study identifies MLB as an active component of Danshen in protecting cardiomyocytes from ischemic injury in both animal and cell models. Further studies reveal this protection effect is mainly due to the inhibition of ischemia-induced p38 activation by MLB. More detailed analysis indicates that MLB does not affect MKK3/6-mediated p38 activation, but specifically inhibits TAB1-mediated p38 activation. Our results not only give light to a new mechanism that might lead to a better understanding of Danshen's therapeutic effect, but also point out TAB1-p38 interaction to be a putative drug target in treating ischemic heart diseases.

\section{ACKNOWLEDGMENTS}

We thank Shun-Mei Xin for her excellent technical support. This research was supported by grants from the National Natural Science Foundation of China (90713047), the Ministry of Science and Technology of China (2008DFB30150), Shanghai Municipal Commission for Science and Technology (08410703500,08431910100, 09PJ1410000), Shanghai Postdoctoral Science Foundation (06R214161), Shanghai Key Laboratory of Signaling and Disease Research (09DZ2260100), and Roche RRDCC Basic Research.

rat myocardium. Circ. Res. 79, 949-956.

Garg, S., Hofstra, L., Reutelingsperger, C. and Narula, J. (2003). Apoptosis as a therapeutic target in acutely ischemic myocardium. Curr. Opin. Cardiol. 18, 372-377.

Garlid, K. D., Paucek, P., Yarov-Yarovoy, V., Murray, H. N., Darbenzio, R. B. D’Alonzo, A. J., Lodge, N. J., Smith, M. A., and Grover, G. J. (1997). Cardioprotective effect of diazoxide and its interaction with mitochondrial ATP-sensitive $\mathrm{K}+$ channels. Possible mechanism of cardioprotection. Circ Res. 81, 1072-1082.

Ge, B., Gram, H., Di Padova, F., Huang, B., New, L., Ulevitch, R. J., Luo, Y., and Han, J. (2002). MAPKK-independent activation of p38alpha mediated by TAB1-dependent autophosphorylation of p38alpha. Science 295, 1249-1250.

Gum, R. J., McLaughlin, M. M., Kumar, S., Wang, Z., Bower, M. J., Lee, J. C., Adams, J. L., Livi, G. P., Goldsmith, E. J., and Young, P. R. (1998). Acquisition of sensitivity of stress-activated protein kinases to the p38 inhibitor, SB 203580, by alteration of one or more amino acids within the ATP binding pocket. J. Biol. Chem. 273 , 15605-15610.

Hu, Z. Y., and Liu, J. (2009). Effects of emulsified isoflurane on haemodynamics and cardiomyocyte apoptosis in rats with myocardial ischaemia. Clin. Exp. Pharmacol. Physiol. 36, 776-783.

Ji, X. Y., Tan, B. K., and Zhu, Y. Z. (2000). Salvia miltiorrhiza and ischemic diseases. Acta Pharmacol. Sin. 21, 1089-1094.

Jiang, R. W., Lau, K. M., Hon, P. M., Mak, T. C., Woo, K. S., and Fung, K. P. (2005). Chemistry and biological activities of caffeic acid derivatives from Salvia miltiorrhiza. Curr. Med. Chem. 12, 237-246.

Jing, Q., Xin, S. M., Cheng, Z. J., Zhang, W. B., Zhang, R., Qin, Y. W., Pei, G. (1999). Activation of p38 mitogenactivated protein kinase by oxidized LDL in vascular smooth muscle cells: mediation via pertussis toxin-sensitive $\mathrm{G}$ proteins and association with oxi- 
dized LDL-induced cytotoxicity. Circ. Res. 84, 831-839.

Kang, J., Shi, Y., Xiang, B., Qu, B., Su, W., Zhu, M., Zhang, M., Bao, G., Wang, F., Zhang, X., Yang, R., Fan, F., Chen, X., Pei, G., and Ma, L. (2005). A nuclear function of beta-arrestinl in GPCR signaling: regulation of histone acetylation and gene transcription. Cell 123, 833-847.

Kim, J. K., Pedram, A., Razandi, M., and Levin, E. R. (2006). Estrogen prevents cardiomyocyte apoptosis through inhibition of reactive oxygen species and differential regulation of p38 kinase isoforms. J. Biol. Chem. 281, 6760-6767.

Li, J., Miller, E. J., Ninomiya-Tsuji, J., and Russell, R. R. 3rd, Young, L. H. (2005). AMP-activated protein kinase activates $\mathrm{p} 38$ mitogen-activated protein kinase by increasing recruitment of p38 MAPK to TAB1 in the ischemic heart. Circ. Res. 97, 872-879.

Liu, J., Shen, H.M., and Ong, C. N. (2000). Salvia miltiorrhiza inhibits cell growth and induces apoptosis in human hepatoma HepG(2) cells. Cancer Lett. 153, 85-93.

Liu, X. H., Xin, H., Hou, A. J., and Zhu, Y. Z. (2009). Protective effects of leonurine in neonatal rat hypoxic cardiomyocytes and rat infarcted heart. Clin. Exp. Pharmacol. Physiol. 36, 696-703.
Ma, X. L., Kumar, S., Gao, F., Louden, C. S., Lopez, B. L., Christopher, T. A., Wang, C., Lee, J. C., Feuerstein, G. Z., and Yue, T. L. (1999). Inhibition of p38 mitogen-activated protein kinase decreases cardiomyocyte apoptosis and improves cardiac function after myocardial ischemia and reperfusion. Circulation 99, 1685-1691.

Rodriguez, M., Lucchesi, B. R., and Schaper, J. (2002). Apoptosis in myocardial infarction. Ann. Med. 34, 470-479.

Tanno, M., Bassi, R., Gorog, D. A., Saurin, A. T., Jiang, J., Heads, R. J., Martin, J. L., Davis, R. J., Flavell, R. A., and Marber, M. S. (2003). Diverse mechanisms of myocardial p38 mitogen-activated protein kinase activation: evidence for MKK-independent activation by a TAB1-associated mechanism contributing to injury during myocardial ischemia. Circ. Res. 93, 254-261.

Thompson, C. B. (1995). Apoptosis in the pathogenesis and treatment of disease. Science 267, 1456-1462.

Wan, J. M., Sit, W. H., Lee, C. L., Fu, K. H., and Chan, D. K. (2006). Protection of lethal toxicity of endotoxin by Salvia miltiorrhiza BUNGE is via reduction in tumor necrosis factor alpha release and liver injury. Int. Immunopharmacol. 6, 750-758.

Wang, W., Peng, Y., Wang, Y., Zhao, X., and Yuan, Z. (2009a). The anti-apoptotic effect of heat shock protein 90 on hypoxia-mediated cardiom yocyte damage through the pi3k/akt pathway. Clin. Exp. Pharmacol. Physiol. 36, 899-903.

Wang, Y. P., Hang, P. Z., Sun, L. H., Zhang, Y., Zhao, J. L., Pan, Z. W., Ji, H. R., Wang, L. A., Bi, H., and Du, Z. M. (2009b). M3 muscarinic acetylcholine receptor is associated with beta-catenin in ventricular myocytes during myocardial infarction in the rat. Clin. Exp. Pharmacol. Physiol. 36, 995-1001.

Wang, X., Morris-Natschke, S. L., and Lee, K.H. (2007). New developments in the chemistry and biology of the bioactive constituents of Tanshen. Med. Res. Rev. 27, 133-148.

Wu, B., Liu, M., Liu, H., Li, W., Tan, S. Zhang, S., and Fang, Y. (2007). Metaanalysis of traditional Chinese patent medicine for ischemic stroke. Stroke 38, 1973-1979.

Wysk, M., Yang, D. D., Lu, H. T., Flavell, R. A., and Davis, R.J. (1999). Requirement of mitogen-activated protein kinase kinase 3 (MKK3) for tumor necrosis factor-induced cytokine expression. Proc. Natl. Acad. Sci. U.S.A. 96, 3763-3768.

Zacharowski, K., Olbrich, A., Otto, M., Hafner, G., and Thiemermann, C. (1999). Effects of the prostanoid EP3-receptor agonists M\&B 28767 and GR 63799X on infarct size caused by regional myocardial ischaemia in the anaesthetized rat. Br. J. Pharmacol. 126, 849-858.

Conflict of Interest Statement: The authors declare that the research was conducted in the absence of any commercial or financial relationships that could be construed as a potential conflict of interest.

Received: 20 June 2010; paper pending published: 09 July 2010; accepted: 03 August 2010; published online: 24 August 2010.

Citation: Du C-S, Yang R-F, Song S-W, Wang Y-P, Kang J-H, Zhang R, Su D-F and Xie X (2010) Magnesium lithospermate B protects cardiomyocytes from ischemic injury via inhibition of TAB1-p38 apoptosis signaling. Front. Pharmacol. 1:111. doi: 10.3389/fphar.2010.00111

This article was submitted to Frontiers in Ethnopharmacology, a specialty of Frontiers in Pharmacology.

Copyright $\odot 2010$ Du, Yang, Song, Wang, Kang, Zhang, Su and Xie. This is an open-access article subject to an exclusive license agreement between the authors and the Frontiers Research Foundation, which permits unrestricted use, distribution, and reproduction in any medium, provided the original authors and source are credited. 